\title{
A Review of Electrodynamic Tethers for Space Applications
}

\author{
Juan R. Sanmartin \\ Universidad Politécnica de Madrid, Madrid, 28040, Spain \\ E.C. Lorenzini ${ }^{2}$ \\ University of Padova, Padova, 35131, Itaty \\ and \\ Manuel Martinez-Sanchez ${ }^{3}$ \\ Massachusetts I nstitute of Technology, Cambridge, Massachusetts, 02139
}

\begin{abstract}
Space applications of electrodynamic tethers, and basic issues and constraints on their operation are reviewed. The status of the bare-tether solution to the problem of effective electron collection from a rarefied magnetized plasma is revisited. Basic modes of tether operation are analyzed; design parameters and parametric domains where a bare electrodynamic tether is most efficient in deorbiting, reboosting, or power generation, are determined. Use of bare tethers for Radiation Belt Remediation and generation of clectron beams for ionospheric research is considered. Tether heating, arcing, and bowing or breaking, as well deployment strategies are discussed.
\end{abstract}

\section{Introduction}

内

The development of tether technology for space has already experienced 18 suborbital and orbital flights beginning with the Gemini-Agena tether experiments in 1967. Eight of those flights used electrodynamic tethers, starting from suborbital flights in 1980. One additional electrodynamic tether system, ProSEDS, was developed and built by NASA but was not launched into space, in 2003, because of unfortunate circumstances.

Electrodynamic tether systems have evolved over the last three decades through the development of different technologies for its main components. Current exchange with the ambient rarefied plasma was soon identified as a technological bottleneck. Early tether systems used a big conductive sphere at the end of the tether as the anode where electrons are collected from the ionosphere, and an electron gun as a cathode to eject those electrons into space at the other tether end. A second option, adopted in one of the tether flights (the Plasma Motor Generator or PMG), consisted in utilizing plasma contactors (hollow cathodes) at both tether ends for either electron collection or ejection.

In the mid 1990s, the bare tether concept was proposed by two authors of this paper to provide a more efficient anode for the collection of electrons. The project ProSEDS was indeed designed and built by NASA and its contractors to test in space the bare tether concept. The goal of that system was to demonstrate the collection of a much highei electron current (than by using a conductive sphere) and, consequently, the generation of a significant Lorentz force to drag down the mother platform, a Delta second stage, to which the tether was attached. ${ }^{2}$

Although bare electrodynamic tethers could be used in a variety of applications with unique benefits, as in operations of Radiation Beft Remediation (RBR), generation of electron beams to produce artificial auroras, or excitation of nonlinear Alfven wavefronts, the fundamental area of application is propellantless transportation. ${ }^{3}$ This can be syntethized conceptually as exchanging momentum (and energy) between a planetary magnetosphere and the tether system, thereby decreasing or increasing the momentum of the tether system. The paper discusses the basic applications in the area of propellanteless propulsion, related to reboosting space platforms, reentering dead satellites and spent stages, and generating electrical power in space. 
Section II presents the basic concepts in conductive space tethers. Results from theory, numerical simulations, and laboratory tests on bare tethers are reviewed in Sec. MI. Design parameters characterizing tether performance are presented in Sec. IV. Drag, Thrust and Power Generation modes of operation are analyzed in Sec. V, RBR and ebeam generation applications are briefly discussed in Secs.VI and VII. Some basic constraints on tether performance are discussed in Secs. VIII (tether heating), IX (tether bowing and breaking), and X (tether arcing). Issues on deployment of tethers are reviewed in Sec. XI. Conclusions are resumed in Sec. XII.

\section{Electrodynamic tether basics}

A non-relativistic transformation relates throughout the electric fields in frames moving with $\mathrm{SC}$ and local ambient plasma,

$$
\bar{E} \text { (tether frame })-\breve{E} \text { (plasma frame })=\bar{E}_{m}=\left(\bar{v}-\bar{v}{ }_{p l}\right) \times \bar{B},
$$

where $\bar{v}, \bar{v}_{p l}$ are the velocities of $\mathrm{S} / \mathrm{C}$ (and tether) and plasma, assumed corotating in the following, and where $\bar{B}$ is the planetary magnetic field, common to both frames. For no significative currents in the ambient plasma, the electric field outside the tether will vanish in the corotating frame; in the tether frame there is then an outside field $\bar{E}_{m}$ that will drive a current $\bar{I}$ inside the tether with $\bar{I} \cdot \bar{E}_{m}>0$ in case of a passive tether system.

The Lorentz force on an insulated tether of length $L$ carrying an uniform current reads $L \bar{I} \times \bar{B}$. With Newton's $3^{\text {rd }}$ law valid for magnetic forces between steady-current systems, a net power loss is seen to hold in the tetherplasma interaction,

$$
L \bar{I} \times \bar{B} \cdot \bar{y}+(-L \bar{I} \times \bar{B}) \cdot \bar{v} p l=-\bar{I} \cdot \bar{E}{ }_{m} L<0,
$$

which is power naturally feeding the tether electric circuit. ${ }^{4}$ The Lorentz force would be drag if $\bar{v}$ is parallel to the SC velocity relative to the plasma, $\bar{v}-\bar{v}_{p l}$, which is the case for prograde, circular equatorial orbits at radius $a<$ ${ }_{4} a_{s}$, where $a_{s}$ is the stationary-orbit radius for the planet considered. For a LEO orbit, and a no-tilt, centered magnetic dipole model ( $B$ northward, $E_{w}$ upward), a typical 'motional' field is $E_{i n}=v B_{\perp} \sim 7.5 \mathrm{~km} / \mathrm{s} \times 0.2$ gauss $=150 \mathrm{~V} / \mathrm{km}$, drag and power reading

$$
\text { Westward Lorentz drag, } F_{M}=L I B_{1}, \quad \text { Drag power, } W_{M}=F_{M} y=I E_{m} L .
$$

In general (for non-zero orbital inclination or a realistic magnetic model) $\bar{B}$ has components in the nrbital plane in addition to the perpendicular component $B_{\perp}$. The relations for current driven by the motional field and drag power keep valid, and the motional field component perpendicular to the orbit $E_{m \downarrow}$ just produces a negligible potential difference across the thin tether, but the perpendicular Lorentz force $F_{M \perp}$ pushes the tether off the orbital plane and may lead to a coupled in-plane/off-plane unstable periodic motion, with the period of the orbit, the growth rate depending sharply on the $\mathrm{SC} /$ tether mass distribution. ${ }^{5}$

\section{Bare electrodynamic tethers}

ED-tether operation requires effective electric contact with the ambient plasma. State-of-art hollow cathodes are indeed effective in establishing cathodic contact: they expel little Xenon along with the electrons and require bias of tens of volts only, thus resulting in negligible contact impedance; alternative cathodes are actively pursued nonetheless. ${ }^{6}$ In turn, the problem of anodic contact with a highly rarefied plasma, which was previously considered to be a bottleneck, was solved in 1992 when it was proposed that, instead of using a big end-collector, the tether be left bare of insulation to allow it to collect electrons over the segment resulting positively polarized, as a giant cylindrical Langmuir probe in the so-called orbital-motion-limited (OML) regime. ${ }^{7}$ Collection can be efficient because the cross-section dimension is thin, while the collecting area is large because the anodic segment may be tens of kilometers long.

The maximum current collected by a round cylindrical Langmuir probe in a quiescent, unmagnetized plasma is the OML current, which for high-bias ratio, $e \Phi_{p} / k T_{\varepsilon}$, reads 


$$
I_{O M L} \approx I_{t h} \sqrt{\frac{4 e \Phi_{p}}{\pi k T_{e}}}=e N_{\infty} \frac{L p}{\pi} \sqrt{\frac{2 e \Phi_{p}}{m_{e}}}, \quad p=2 \pi R,
$$

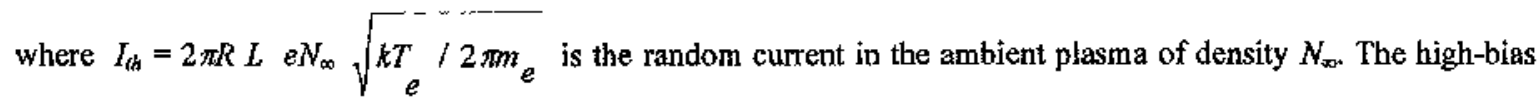
OML law, when valid, is robust, the ratio $I_{\mathrm{OML}} / I_{\mathrm{th}}$ being independent of both Debye and temperature ratios, $R / \lambda_{\text {De }}$ and $T_{i} / T_{e}$; of the ambient ion distribution function; and of the ambient electron distribution if isotropic. Its domain of validity is given by a single condition

$$
R \leq R_{\max } \equiv \lambda_{D e} \widetilde{R}_{\max }\left(e \Phi_{p} / k T_{e}, T_{i} / T_{e}\right) .
$$

For a typical $T / T_{e}$ ratio about unity, and the high-bias values of interest for tethers, $R_{\max }$ is close to the Debye length $\lambda_{D e}{ }^{8}$ For $R \geq R_{\text {max }}$, the current reads

$$
\frac{I}{I_{O M L}(p)}=G\left(\frac{R}{\lambda_{D e}}, \frac{e \Phi p}{k T_{e}}, \frac{T_{i}}{T_{e}}\right) .
$$

with the function $G$ weakly dependent on bias, and roughly depending on a single variable, $R / \lambda_{D e}$. $\widetilde{R}_{\max }\left(T_{i} / T_{e}\right)$.

Excellent detailed agreement is found in work to appear between analytical (asymptotic) results and numerical results from both steady-state Vlasov calculations ${ }^{10}$ and particle-in-cell simulations: ${ }^{11}$ The potential $\Phi(r)$ behaves as $\Phi \sim \ln r$ in a large probe neighbourhood free of space-charge effects because of the high bias, and as $\Phi \sim 1 / r$ faraway, while, in between, $r^{2} \Phi$ presents a minimum at a point outside the sheath, and a maximum at a point $m$ well inside. The density itself shows a minimum at point $m$, and a maximum closer to the probe. Agreement is

* found for $R$ close to, but below $R_{\text {max }}$, on values, and positions, of density minimum and maximum; on sheath radius; and on the $\Phi \sim \ln r$ behaviour around the probe. There is also agreement on broad parametric laws for sheath radius, density minimum, and current beyond the OML regime. ${ }^{12}$

Note that a tether is a singular Langmuir probe because bias and current will vary along its length, but the extremely large length-to-radius ratio allows using uniform-bias results at each local tether section. Both $F_{M}$ and $W_{M}$ now involve the current $I_{a v}$ averaged over the tether length. In the absence of ohmic effects, to be discussed in Sec.IV, the tether is equipotential in its own frame, whereas the potential outside varies linearly at the $E_{m}$ rate; the local bias then decreases linearly with distance from the anodic end, from a maximum $E_{m} L$ there to (near) zero at the hollow cathode. If collection is everywhere OML, one finds

$$
I_{a v, O M L}=\frac{2}{5} e N_{\infty} \frac{L p}{\pi} \sqrt{\frac{2 e E_{i n} L}{m_{e}}} .
$$

A figure of merit for the Lorentz drag $L I_{\pi v}$, OML $B_{\perp}$ on a tether would be its ratio to the drag by neutrals on a body of equal front-area $A_{\perp}=2 R L$, as given by a standard aerodynamics law. That ratio reads

$$
\frac{L L_{a v, O M L}{ }^{B}}{C_{D^{A} \perp} \rho v^{2} / 2} \equiv \frac{4 \sqrt{2}}{5 c_{D}} \sqrt{\frac{m_{i}}{m_{e}}}\left(\frac{\Omega_{i} L}{v}\right)^{3 / 2},
$$

where the drag coefficient $C_{D}$ is typically of order unity, and we set $\rho=m_{1} N_{\infty}$. The ratio above is usually an extremely large number. For oxygen, the square root of the ion-to-electron mass ratio is about 171 . For $L=10 \mathrm{~km}$, and typical LEO values, ion-gyrofrequency $\Omega_{l}-200 / \mathrm{s}$ and $y \sim 8 \mathrm{~km} / \mathrm{s}$, Eq. (6) gives a ratio of order of $10^{6}$.

The OML current law (3) is also valid for any arbitrary convex cross-section shape with $p$ its actual perimeter ${ }^{13}$ Laws (4) and (5) are valid too, except that certain equivalent radius $R_{e q} \neq p / 2 \pi$ must be calculated and used to replace $R$. Because of the high bias, determining $R_{e q}$ for each convex cross section just requires solving the Laplace equation between an arbitrarily large circle and the cross section, at its center, at bias $\Phi_{\mathrm{p}}{ }^{14}$ For a thin tape or foil one finds $R_{e q}=p / 8$. Note that a thin foil, which collects the same OML-current as a round wire of equal cross-section perimeter, will be much lighter, the optimal tether thus presenting three disparate dimensions, $L \gg$ $p / 2$ (foil width) $\gg h$ (foil thickness). 
Further, the law (3) still holds to great accuracy for concave shapes, for which we would have $I<I_{O M L}(p)$ irrespective of cross section size, if the actual perimeter is replaced by the perimeter $p_{\text {ef }}$ of the minimum-perimeter cross-section envelope. ${ }^{14}$ Also, multiple numerical calculations and laboratory tests have been carried out in trying to determine whether all above results would hold in a flowing magnetized plasma, at the mesothermal speed of a LEO orbit, which does not break the ambient electron-distribution isotropy, and for ambient electron gyroradius well above both $R_{\text {er }}$ and $\lambda_{D e}{ }^{15-17}$ The Japanese Space Agency JAXA will test bare-tether collection in a suborbital flight in August 2009 . $^{\text {is }}$

\section{Tether circuit parameters}

In addition to the basic ratios for the collection law, $R_{\mathrm{pq}} / R_{\max }, p_{\text {eq }} / R_{e q}$, there is a number of dimensionless design parameters in the electric tether circuit affecting performance. In absence of an active power system, the current will be limited by its short circuit value, $\sigma_{c} E_{m} A$, arising from the tether resistance $Z_{q} \equiv L / \sigma_{\mathrm{r}} A$. A characteristic length $L^{*}$ gauges ohmic effects for bare tethers, ${ }^{7}$

$$
\begin{aligned}
& \frac{4}{3} e N_{\infty} \frac{L^{*} p}{\pi} \sqrt{\frac{2 e E_{m} L^{*}}{m_{e}}}=\sigma_{c} E_{m} A=\frac{E_{m} L}{Z_{t}}, \\
& L^{*} \propto E_{m}^{1 / 3}\left(\frac{\sigma_{c} A}{N_{e} p}\right)^{2 / 3} .
\end{aligned}
$$

In general, the average current can be written as

$$
\frac{I_{a v}}{\sigma_{c} E_{m} A} \equiv i_{a v}\left(\frac{J_{a v, O M L}}{\sigma_{c} E_{m}^{A}}\right)
$$

with $I_{a v}$, om given by Eq. (6), and $i_{a v}$ actually a function of $L / L^{*}$, as following from the identity

$$
\frac{I_{a v, O M L}}{\sigma_{c} E_{m} A}=\frac{3}{10}\left(\frac{L}{L}\right)^{\beta / 2}
$$

$I_{a v}$ approaches $I_{a v, O M L}$ at vanishing $L / L^{*}$ and dimensionless current $i_{a v}$, and approaches $\sigma_{c} E_{w r} A$ with $i_{s v} \sim 1$ at large $L / L^{*}$. Collection performance per unit tother mass would be optimum for $L \gg L^{*}$, when current attains its maximum, short-circuit value. Since length $L^{*} \propto R^{2 / 3}$ for round-wire tethers, and $L^{*} \propto h^{2 / 3}$ for a thin foil of thickness $h$, as seen in (9) above, moving from round wires to foils improves performance as already pointed out in Sec. III. Notice that a large ratio $L / L^{*}$ is equivalent to a negligible contact impedance for bare-tether collection.

For power generation, the tether circuit involves both the ratio $L / L^{*}$, and a second ratio, $Z_{l} / R_{t}$, where $Z_{l}$ is the impedance characterizing a power load, located at the cathodic end between tether and hollow cathode. Some value $i_{q v}\left(L / L^{*}, Z_{j} / R_{t}\right)<1$ would now yield a maximum of the generator efficiency $\eta_{g}$, which is the ratio $Z_{l} I_{C}{ }^{2} / I_{m} E_{m} L$, with the current at the hollow cathode $I_{C}>I_{\text {aw }}$

In order to invert current direction and both anodic and cathodic end locations, and produce thrust, a power source, and the hollow cathode, must lie at the top, with electrons flowing upwards (for eastward orbits in LEO). The dimensionless current $i_{\pi v}=I_{a v} \sigma_{i} E_{m} A$, now depends on $L / L^{*}$ and $W_{e} / \sigma_{\varepsilon} E_{m}^{2} A L \quad\left(W_{\varepsilon}=\right.$ electric power supplied), and on a third parameter, $L_{i} / L$, where $L_{j}$ is the length of a tether segment next to the top that must be insulated. This is because a fuily bare tetber would have low efficiency, as the electrons collected near the top, where bias would be largest, would do little push work but would consume energy from the power source.

The ratio between a dimensional parameter for the tether,

$$
\frac{p}{\sigma_{c} E_{m}^{2}} \approx 3.43\left(\frac{150 \mathrm{~V} / \mathrm{km}}{E_{m}}\right)^{2} \frac{\mathrm{kg}}{\mathrm{kw}} \quad \text { (for aluminum), }
$$


and the inverse specific power $\alpha(\mathrm{kg} / \mathrm{kw})$ of a power source, be solar-array, fuel-œell, or Radioisotope Thermal Generator, will also appear in the tether mass budget. Finally, effects from the magnetic self-field, due to the tether current itself, increase with a dimensionless parameter $K_{s}$ that is proportional to $R_{i}^{5 / 3}$ for round wires, and to $h_{f}^{2 / 3} \times$ widih for foils. They appear always negligible for foils under any reasonable tether / ambient conditions. ${ }^{19}$

\section{Tether operation modes}

\section{A. Deorbiting mode}

Probably the most obvious application of an electrodynamic tether is as end-of-life system to reenter dead satellites and spent rocket stages, which will increasingly crowd the LEO region. This system, with different system names, has been studied by various authors in sufficient detail. The alternative device would be Ion Thrusters powered by solar arrays..$^{20} \mathrm{~A}$ figure of merit for comparing these alternatives, in order to identify regions in the system parameter space in which an electrodynamic tether may be more desirable would be the ratio between required drag-system mass $M_{d}$ and deorbiting impulse $F \tau$ that either system must provide within some altotted time $\tau$.

For the Ion Thruster, where $M_{d}$ is basically made of propellant consumed and power plant, that ratio reads

$$
\frac{M_{l d}}{F \tau}=\frac{\dot{m} \tau+\alpha \dot{m} c^{2} / 2 \eta_{I}}{\dot{m} c \tau}=\frac{1}{c}+\frac{\alpha c}{2 \eta_{l} \tau},
$$

where $\dot{m}, c$, and $\eta_{l}$ are Thruster mass flow rate, jet velocity, and propulsive efficiency (a typical value being 0.65 ). Values of the specific power $\alpha$ of a solar-power plant are typically a few tens of $\mathrm{kg} / \mathrm{kW}$ in case a dedicated plant is required, and $\alpha$ less than $10 \mathrm{~kg} / \mathrm{kW}$ if the plant is independently in place. We shall consider the optimal Thruster, for which the jet velocity makes minimum the ratio in (12) for given $\alpha$ and time $\tau$,

$$
\left.\frac{M_{I d}}{F_{\tau}}\right|_{\min }=\frac{2}{c_{o p t}}=\sqrt{\frac{2 \alpha}{\eta_{I} \tau}} .
$$

For a tether, $M_{d}$ would just be $\alpha_{t}$ times tether mass $m_{t}$, with the factor $\alpha_{t}>1$ accounting for both tether and basic tether-related hardware. We would then have

$$
\frac{M_{t d}}{F \tau}=\frac{\alpha_{t} \rho A L}{L_{a v} B_{\perp} \tau}=\frac{v \alpha_{t} \rho}{i_{\alpha v}\left(L / L^{*}\right) \tau \sigma_{c} E_{m}^{2}} .
$$

From Eqs. (13) and (14) we find

$$
\frac{M_{l d}}{M_{I d, \min }}=\frac{\sqrt{\eta}}{i_{a \nu} \tilde{E}_{m}^{2}} \sqrt{\frac{\nu^{2} \alpha}{2 \tau}} \approx \frac{\sqrt{\eta_{I}}}{i_{a \nu} \tilde{E}_{m}^{2}} \sqrt{\frac{3.26 \text { days }}{\tau}},
$$

where we used representative values for LEO velocity $\nu=7.5 \times \mathrm{km} / \mathrm{s}$ and mid-range $\alpha=10 \mathrm{~kg} / \mathrm{kW}$, and introduced a dimensionless motional field

$$
\tilde{E}_{m} \equiv E_{m} \sqrt{\alpha \sigma_{c} / \alpha_{t} \rho},
$$

with values just above (below) unity for $\alpha_{i} \sim 2-2.5$, at the high (low) ends of LEO motional fietd and $\alpha$ ranges. The ratio in (15) reaches its minimum at large $L / L^{*}$ when $i_{a v}$ approaches unity. Clearly, the tether will beat the Ion Thruster except for short missions, unless $L / L^{*}$ is small.

\section{B. Thrust mode}

In the thrust mode, with tethers as well as Ion Thnusters powered, a power-subsystem mass $\alpha W_{c}$ must be added to $M_{d d}$. We then have

$$
\frac{M_{t T}}{F \tau}=\frac{\alpha_{t} \rho A L+\alpha W_{e}}{L_{a v} B_{\perp} \tau}=\frac{M_{t d}}{F \tau}\left(1+\frac{\alpha W_{e}}{\alpha_{t} m_{t}}\right),
$$

with $W_{e}=F_{V} / \eta_{t}$ where $\eta_{t}$ is the tether propulsive efficiency, and 
Using Eqs. (13), (14) and (17) we find

$$
\frac{\alpha W_{e}}{\alpha_{t} m_{t}}=\tilde{E}_{m}^{2} \frac{W_{e}}{\sigma_{c}{ }^{E}{ }_{m}^{2} A L} \text {. }
$$

$$
\frac{M_{t T}}{M_{I T, \min }}=\frac{\sqrt{\eta_{I}}}{i_{a v} \widetilde{E}_{m}^{2}}\left(1+\widetilde{E}_{m}^{2} \frac{i d v}{\eta_{t}}\right) \sqrt{\frac{v^{2} \alpha}{2 \tau}} .
$$

Both $i_{a v}$ and efficiency $\eta_{i}$ are complex functions of design ratios $L / L^{*}, L / L$, and $W_{e} / \sigma_{c} E_{m}{ }^{2} A L$. At large $L$ $/ L^{*}$, minimum tether-system mass occurs at $L-L_{i} \sim L^{*}$, and $W_{e} / \sigma_{c} E_{m 1}{ }^{2} A L$ such that bias at tether bottom is negligible. ${ }^{21}$ This would correspond to values

$$
\alpha W_{e} / \alpha_{t} m_{t} \approx 1+\widetilde{E}_{m}, \quad i_{a v} \widetilde{E}_{m} \approx 1, \quad \eta_{t}\left(1+\widetilde{E}_{m}\right) \approx \widetilde{E}_{m} .
$$

For $\widetilde{E}_{m} \approx 1$, the ratio in Eq. (19) is 3 times larger than the ratio in (15). Tethers would beat Ion Thrusters for mission times beyond about 20 days.

A case where tethers would compete with rockets rather than Ion Thrusters, concerns reboosting the International Space Station. A study has shown that a bare electrodynamic-tether system of moderate length could save, under conservative assumptions, about $80 \%$ of the chemical propellant used for reboosting of the station over its lifetime. ${ }^{22}$

\section{Power generation mode}

Fuel cells typically serve as primary power source for 1-2 weeks, while batteries serve for very short missions, say less than one day, and solar arrays for long missions. It turns out that, in absence of solar power in LEO, a combination ED-tether/rocket, with the tether providing electric power and the chemical rocket providing thrust to compensate the magnetic drag on the tether, is more mass-efficient than a fuel cell. ${ }^{23} \mathrm{~A}$ tether in the generation mode provides an useful power $W_{\epsilon}=\eta_{g} W_{M}\left(W_{M}=F_{M} v\right)$. Since thrust by the rocket $\dot{m}_{r k}{ }_{r k}{ }_{r k}$ must balance the drag $F_{M}$ to keep the SC velocity, there follows a relation $W_{M}=\dot{m}_{r k} c_{r k} v$ that is definitely greater than the rocket power output, ${ }^{3 / 2} \dot{m}_{r k}{ }^{c} r k{ }^{2}$ by a factor $2 v / c_{r k}>3$ for $v \approx 7.5 \mathrm{~km} / \mathrm{s}$, and $c_{r k} \approx 4.5 \mathrm{~km} / \mathrm{s}$ for the LOX-LH rocket having the greatest jet velocity. It can be shown that the excess power in the combined tether/rocket system arises from the SC energy decrease due to the rocket fuel-mass loss. ${ }^{24}$

Fuel cells being lighter, however, the tether/rocket combination only saves mass for a long enough time $\tau$,

$$
\dot{m}_{f c} \tau>\alpha_{t} \rho A L+\dot{m}_{r k} \tau,
$$

both systems providing equal power $W_{b}$

$$
\eta_{f c}{ }^{\dot{m}} f c{ }_{f c}^{v}{ }^{2} / 2=W_{\mathrm{p}}=\eta_{g}{ }_{r k}{ }_{r k}{ }^{\nu} \text {. }
$$

The energy per unit fuel mass liberated in a LOX-LH $\mathrm{H}_{2}$ cell is about $1,25 \times 10^{7} \mathrm{~J} / \mathrm{kg}$, which we write as $1 / 2 v_{f \varepsilon}^{2}$ with $v_{f o} \approx 5 \mathrm{~km} / \mathrm{s}$, and the fuel cell efficiency is about $\eta_{j c} \sim 0.75$. It follows from Eqs. (21) and (22) that the tether/rocket system is lighter for times satisfying

$$
i_{a v}\left(\eta_{g}-b\right) \tau>\frac{\alpha_{t} \eta_{f c} \rho v_{f c}^{2}}{2 \sigma_{c} E_{m}^{2}} \quad\left(b=\frac{\eta_{f c} v_{f c}^{2}}{2 c_{r k} v} \approx 0,28\right) .
$$

Minimum mass correspond to maximum $i_{a w} \times\left(\eta_{g}-b\right)$. In general, $i_{a v}$ and $\eta_{g}$ vary oppositely with $Z / Z_{i}$; in particular, at large $L / L^{*}$, we have $i_{a v} \approx 1-\eta_{g}$, that maximum occurring at $\eta_{g}=0.64, \quad i_{w} \approx 0.36$. The tether/rocket system would be lighter than a fuel cell for ${ }^{24}$

$$
\tau>2.9 \alpha_{\mathrm{f}}\left(150 \mathrm{Vkm}^{-1} / E_{\text {tr }}\right)^{2} \text { days. }
$$




\section{Radiation Belt Remediation}

The RBR problem arises from the possibility of man-made intensification of the already dangerous levels of high-energy particle populations trapped in the geomagnetic field in the altitude range of $1,000-15,000 \mathrm{~km}$. These particles, typically protons and electrons, have pitch angles with respect to the magnetic field high enough to induce repeated reflections above the dense atmosphere altitude. Any intervention that can reduce this pitch angle can ultimately lead to absorption of the particle as it dips farther into the atmosphere near magnetic poles.

Among the possible mechanisms that have been proposed, two would involve in-situ use of tethers.

1) Whistler wave injection in the magnetospheric cavity to mimic the diffusive scattering of ionospheric wave hiss that constitutes a natural decay mechanism for the trapped particles.

2) Scattering of particles by the high potential structure around a tether or combination of tethers, which would send a fraction of the ions into the loss cone for thejr magnetic line.

In the original work for the second mechanism, ${ }^{25}$ a pair of tethers would be biased in a double-probe arrangement, with most of the potential deviation from the background being taken up by the negative tether, which then becomes the active scattering center. Since the trapped proton energy is up to $1 \mathrm{MeV}$, the bias must also be of this order. The electron temperature in the background magnetospheric plasma is of the order of $100 \mathrm{eV}$, while the plasma at the bigh altitudes considered could be as low as $10^{3} / \mathrm{m}^{3}$, giving a Debye length $\lambda_{D e}$ of about $20 \mathrm{~m}$.

For a round-wire tether of radius $R$ certainly very sinall compared with $\lambda_{D \mathfrak{p}}$, a law for the sheath radius $r_{s h}$

$$
\frac{e{ }_{p}}{k T_{e}}=k\left(\frac{r_{s h}}{\lambda_{D e}}\right)^{4 / 3} \ln \frac{r_{s h}}{R},
$$

with a factor $k \sim 1.3-1.5$ weakly dependent on the ratio $r_{s h} / \lambda_{D_{e}}$ was obtained both numerically ${ }^{10}$ and analytically. ${ }^{12}$ For $R$ in the milimeter range, Eq. (25) gives a large sheath radius of up to $2 \mathrm{~km}$. Scattering is modelled through a Coulombic (logarithmic) potential, arising from the extremely high bias, reaching down to vanish at the sheath radius. Classical scattering formulation determines condition for the loss cone (defined as the pitch range allowing penetration of the particle below $110 \mathrm{~km}$ altitude in either polar region).

Considerable design issues are involved through conditions of zero Lorentz force, small Lorentz torque, and low electron collection and power requirements. ${ }^{26}$ Multiple connected tethers involve interested dynamics. ${ }^{27}$

\section{Auroral effects from tethers}

A bare tether over $10 \mathrm{~km}$ long, left electrically floating (i.e. using no electric contact devices at the tether ends), in Low Earth Orbit, would be an effective electron-beam source to produce artificial auroras. Because current will vanish at both ends and the ion-to-electron mass ratio is large, the tether would be biased highly negative and attract ions over most of its length. Ions impacting with $\mathrm{keV}$ energies would liberate secondary electrons, which would locally accelerate away from the tether, then race down geomagnetic lines and result in auroral emissions in the ionospheric E-layer. Beyond auroral effects proper, observations down the beam from the spacecraft carrying the tether could provide real-time mapping of density profiles for dominant neutral species in the lower thermosphere, of interest for numerical simulations of the atmosphere lying below and in reentry predictions. ${ }^{28}$

Since 1969, electron-beam sources on board spacecraft (S/C) have been used in active experiments for producing artificial auroras but such "standard" e-beams are marred in several ways. Beam-firing affects the S/C potential serving as ground for the beam source. Gross perturbations produced by the intense emission in plasma around the $\mathrm{S} / \mathrm{C}$ affects the beam itself, and the luminous glow arising from electron bombardment in the return current contaminates optical instruments. Beam flux up to two orders of magnitude greater than in the strongest natural auroras compensates for the thinness of the emitting layer and makes ground observation possible; high-flux beams, however, produce hot electrons and plasma fluctuations, and are distorted by nonlinear plasma interactions.

The tether e-beam is free of $\mathrm{S} / \mathrm{C}$ charging, and from plasma-interaction problems, beam density and flux being low, and beam emission takes place far from any instrument. The tether low-flux, thin beam, however, exhibits brightness for ground observation as low as 1 Rayleigh, light sources in the night sky masking such signals. On the other hand, brightness is much greater for observation from the spacecraft, over $10^{2}$ Rayleigh for prominent bands and lines, also allowing continuous measurements (brightness is roughly proportional to both tape width and length). This is impracticable for the thin cross section of a standard beam, but the tether beam has a cross section dimension of order of the tether length, or about $10^{4}$ meters.

Each point in the tether, which is deployed downwards, emits monoenergetic secondary electrons, but both electron flux and energy increase near linearly with distance $s$ from the top of the tether. Observations from the 
spacecraft along any straight line at angle $\psi$ from the magnetic field direction, covering some altitude range $\Delta z$ over the emission region, would mix $s / z$ effects. The narrow footprint of the beam in the emitting layer will then show a peak in brightness versus angle $\psi$, about $6^{\circ}$ wide allowing tomographic analysis of the signal.

\section{Tether heating}

The energy equation determining the temperature $T(s)$ of an element of tether length $\delta s$ is

$$
w h \delta s \rho c_{t} \partial T / \partial t=\dot{w}_{t h} \delta s-2 w \delta \varepsilon_{t} \sigma_{B}{ }^{4}
$$

where $c_{f}$ and $\varepsilon_{1}$ are tether specific heat and emissivity respectively, and $\sigma_{B}$ is the Stefan-Boltzmann constant. We are ignoring any low heating from the natural environment. The tether thermal balance in Eq. (26) is a local balance because the thermal diffusivity for aluminum is of order $10^{-4} \mathrm{~m}^{2} / \mathrm{s}$, which is many orders of magnitude smaller than any reasonable value for $L^{2} t_{m}$, where $t_{t h}$ is a characteristic time for changes in ambient or tether conditions.

The heating power in (26) is made of two contributions,

$$
\dot{w}_{t_{h}} \delta s=\frac{I^{2} \delta s}{\sigma_{c} w h}+\Delta V \frac{d I}{d s} \delta s,
$$

one due to ohmic dissipation from the curtent $I(s)$ flowing through the element $\delta$ s, and a second contribution due to the energy brought up to the element by the impact of collected electrons. The first term, rewritten as $i \sigma_{c} h w \delta E_{\mathrm{m}}{ }^{2}$, would give a characteristic heating rate, ignoring the radiation cooling term in (26),

$$
\frac{\partial T}{\partial t}=i^{2} \frac{\sigma_{c} E_{m}^{2}}{c_{t} \rho} \approx 19.7 i^{2}\left(\frac{E_{m}}{150 \mathrm{~V} / \mathrm{km}}\right)^{2} \frac{K}{\min u t e} .
$$

* For a tape thin and long enough that the ohmic limited current, $i \approx 1$, is attained somewhere, heating appears to be fast enough that radiation would result in a limit temperature,

or

$$
T^{4}=i^{2} \sigma_{c} E_{m}^{2} h / 2 \varepsilon \sigma_{B}
$$

$$
T \approx 137 \mathrm{~K} \frac{\sqrt{i}}{1 / 4}\left(\frac{h}{0.05 \mathrm{~mm}}\right)^{1 / 4}\left(\frac{E_{\mathrm{m}}}{150 \mathrm{~V} / \mathrm{km}}\right)^{1 / 2} .
$$

The heating rate in (28) is independent of tether cross-section dimensions, whereas the limit temperature in (29) is lower for a foil than for a wire of equal perimeter (though the wire may reach $i$ values well below unity). For $h=$ $0.05 \mathrm{~mm}, E_{\mathrm{w}}=150 \mathrm{~V} / \mathrm{km}$, and $\varepsilon_{\mathrm{i}}$ as low as 0.2 , Eq. (29) shows that the foil keeps cool, at $T \approx 205 \mathrm{~K}$.

For $L \ll<L^{*}$, with negligible ohmic effects, the second contribution to the heating power in $(27)$ would be maximum at the anodic end, where bias would be greater, would read

$$
w_{t h} \delta s=E_{m} L \times e N_{\infty} \frac{2 w \delta s}{\pi} \sqrt{\frac{2 e E_{m} L}{m_{e}}} .
$$

Setting above $L \rightarrow L^{*}$ one just recovers heating of the order of the first term in (27) for $i$ of order unity.

\section{Tether bowing and breaking}

Avoiding too large tether bowing due to the strong lateral Lorentz force at capture may require a corresponding strong tension, which might require tether spinning. For a simple, conservative estimate of bowing consider the 
classical equation of statics for a rope under lateral load per unit length $q$ and supported at the two ends, with the tension assumed large enough to keep deflections small,

$$
d^{2} y / d s^{2}=q / F_{3}
$$

where $y$ is the lateral deflection of the rope and $F_{T}$ the tensile force. Here, the distributed lateral load $q(s)$ is the Lorentz force per unit tether length,

and $s$ measured from the anodic end as previously.

$$
q(s) \equiv \delta F / \delta s=B I(s),
$$

In the ohmic-dominated limit we would have $q \propto I=\sigma_{c} E_{m} w h=$ const over near the entire tether length. Integrating Eq. (30) twice with boundary conditions $y(0)=y(L)=0$, yields the classical result for uniform load distribution, with maximum deflection

$$
\mid V_{\max } / L=F / 8 F_{T} \quad \text { (at mid-point, } s=L / 2 \text { ). }
$$

The deflection in the opposite limit of weak ohmic effects is similar. A straightforward integration of Eq. (30) using the corresponding current law $F(s)$, yields a maximum deflection (at $s \approx 0.564 L)|y|_{\max } / L=F / 7.6 F_{T}$.

The gravity-gradient force provides the tension required to keep a tether taut in circular orbit around a planet in situations with small lateral loads. The standard formula for the gravity-gradient tensile force on a tether kept straight along the local vertical while orbiting under negligible lateral force is

$$
3 \omega_{p j}^{2} L M_{S C} / 4
$$

where a massless tether and equal masses $M_{S C} / 2$ at either end are considered. Two thirds of the tension in (33) arise from the fact that gravitational forces decrease as the inverse square of distance from the planet center. Taking into account tether mass, with equal end masses $\left(M_{S C}-m_{t}\right) / 2$, yields a small correction that makes the tension vary with distance $s^{*} \equiv(L / 2)-s$ from mid-tether,

$$
F_{T} \approx \frac{3 \omega p^{2} L m}{4}\left[\frac{M_{S C}}{m_{t}}-\frac{2}{3}\right],
$$

where we finally average over the tether length for simple estimates. Here we shall ignore the second term in the bracket above.

For a long, light-tape tether, for which Eq. (32) might apply, we would have

$$
\frac{|y|_{\max }}{L}=\frac{F}{8 F_{T}}=\frac{{ }_{a v} \sigma_{c} E_{m} A L B \perp}{{ }^{6 \omega_{p l} L \rho A L}} \frac{m_{t}}{M_{S C}},
$$

with $i_{a v}$ close to unity in the Lorentz force. Using Eq. (1I) and values $M_{S C} \sim 30 \mathrm{~m}, L=20 \mathrm{~km}, v=7.5 \mathrm{~km}$, and the Earth rotation rate, yields a bowing ratio greater than unity.

Setting the tether to spin with $\omega_{t}^{2} \gg 3 \omega_{p l}^{2}$ can provide the tension required to keep lateral bowing small. The tension under tether spin is obtained from Eq. (34) itself through the simple replacement $3 \omega_{p l}{ }^{2} \Rightarrow \omega_{t}{ }^{2}$, neglecting the gravity-gradient contribution. A tether spin $\omega_{t} \sim 10 \omega_{p l}$, for 2.5 hour spin period, would make bowing small.

The tensile stress $\sigma_{T}$ reads

$$
\sigma_{T}=\frac{F_{T}}{w h} \approx \frac{{ }^{o}{ }_{t}^{2}{ }^{2} \rho}{4} \frac{M_{S C}}{m_{t}} .
$$

For $M_{S C}=30 m_{1}$ and a $20 \mathrm{~km}$ aluminum foil, at 2.5 hours spin, Eq. (62) yields

$$
\sigma_{T} \approx 4 \times 10^{6} \mathrm{~N} / \mathrm{m}^{2} \text {, }
$$

which is well below the tensile strength of aluminum.

\section{Arcing in tethers}


In a plasma with no high energy charges, as in LEO, arcing may occur at triple points, which are junctions where conductor, dielectric, and ambient plasma are present. The critical case is where the conductor is biased highly negative relative to the ambient plasma. This may be of particular interest for tethers, an example being the tether prepared for NASA's ProSEDS mission, planned for and aborted in 2003. ProSEDS had $10 \mathrm{~km}$ of nonconductive material and $5 \mathrm{~km}$ of aluminum. A $200 \mathrm{~m}$ segment of this conductive tether, next to the Deployer/Canister carried an insulted coating.

There were 2 triple points: One at the transition between conductive (bare) / and nonconductive segments; this would be positively biased relative to the plasma. No arcing was found in tests. There was a second triple point at the transition between conductive (bare)/ and conductive (insulated) segments; bias could be negative in the operational scheme. Arcing did occur when bias was negative enough. Arcing did also occur at pinholes (breaches of the insulation) arranged for testing. Arcing mitigation at the above triple point involved partial covering with a semiconductor sleeve. ${ }^{29}$

Arcing in GEO, where high encrgy electrons may be present, might exhibit quite different phenomena: Snapover and flashover, both related to the fact that insulators may have high secondary electrons yield. A snapover involves again three elements, plasma $a_{3}$ a positively charged conductor, and an insulator surface next to it. Attracted electrons missing the conductor may hit the insulator. Secondary electrons emitted by the insulator are collected by the conductor. In this way, a collecting surface greater than the conductor surface itself there results. The surface of the insulator is said to be 'snapped over'. Current may be much greater than in absence of the insulator, and the bombarded surfaces may glow if the plasma density is high enough, and may lead to permanent damage.

Flashover may occur after a magnetic substorm, a conductive surface in a spacecraft in GEO then being charged negative by energetic electrons. On the other hand, an insulator next to the conductor might be charged positive by the intense electron secondary emission due to the incident electrons. This condition is called an "inverted potential gradient". As the potential difference reaches a threshold, an electrostatic discharge (ESD) through breakdown in the insulator may occur. In turn, this triggers a "blow-off" discharge between conductor and space plasma. A 'flashover' discharge then occurs as the now negatively charged plasma travels over the insulator neutralizing its original positive charge.

*

\section{Tether Deployment}

Strategies for tether deployment are determined by orbital conditions and the desired state of the tether at the end of deployment. For a tether system that, once deployed, has to be aligned with the local vertical, a libratingdeployment strategy will likcly,be implemented, whereas, for a system that, once deployed, requires spinning, a spinning deployment strategy will be most suitable. In the former case, a relatively strong gravity gradient (e.g., in LEO) is required to keep the tether straight and bound to librate (or stay aligned) with the local vertical. In the latter case, the gravity gradient is not an issue and in several applications may actually be a disturbance to the dynamics of the spinning system.

Flight-mission tether deployments have thus far taken place in LEO following the librating-deployment strategy. In librating deployment the system does not spin but rather it librates about its local-vertical stable position determined by the gravity gradient; the tether tension may need to be maintained in the early stages of deployment by, for example, a thruster firing along the tether line. One can reduce the time spent at short tether length by ejecting the daughter satellite at relatively high speed and very low tension in close to free-flying conditions. Subsequently the deployer will start braking on the tether once a significant tether length has been reached and the gravity gradient forces are taking over.

In a spinning deployment, the centrifugal forces generated by the spin produce a tether tension that facilitates the extraction of the tether from the deployer. Spinning deployment can be faster than a librating deployment because there are no limits imposed on the tether exit velocity by the maximum amplitude of a stable libration. Spinning deployments have not been experimented in space yet but they have been studied by several authors and their realization is expected to be straightforward. They can take place in deep space or more generally in conditions of weak gravity gradient. ${ }^{30,31}$

In a possible scenario, the two end masses are separated initially at a relatively low speed, the system is then put into a spin by lateral thrusters placed on the end masses, and deployment proceeds with the help of centrifugal forces while the tether tension is controlled by a braking mechanism that lets the tether out. Alternatively, the end masses could be separated at high speed by exceeding the condition of librational stability and hence forcing the system to tip over. Lateral forces will still be needed to keep the spin going at the desired spin rate as the tether length and the moment of inertia of the system increase.

Tether deployment can use passive (non-motorized) or active (motorized) deployers. Active deployers must be used for tethered systems that need to be retrieved (e.g., TSS missions) or for systems in which the tether length 
must be varied during the mission. If neither retrieval nor length control are involved, a passive deployer, which is much lighter and simpler, is normally well suited for deploying the tether and keeping its length stationary (e.g., SEDS and PMG missions).

The other distinguishing factor for deployers is their mechanics They can utlize a drum to reel out and in (if necessary) the tether which is spooled on the reel. If it is necessary to reel in the tether then a leveling mechanism is required to distribute the tether evenly along the axis of the drum. Passive deployers use a stationary spool whereby the tether unravels from the spool by pulling it out along its axis. Such deployers (e.g., the SEDS deployer) can not reel in the tether but they are very simple and light. Moreover, in a stationary spool the only moving mass is the exiting portion of the tether mass and, consequently, the tether can recejve sudden accelerations without incurring very high values of tension. On the contrary, in a reeling deployer the whole moment of inertia of the drum and the spool is accelerated and strong tensions are unavoidable with strong accelerations. This consideration implies that a spring ejection system that imparts a strong initial acceleration is not suitable in conjunction with a reeling deployer but it is for a stationary-spool deployer.

In case of a wide tape, a reeling system secms preferable to a stationary spool because the tape could be twisted while exiting along the axis of the stationary spool and would be likely to produce high friction or even to cause jamming. The Atex deployer was designed to deploy a 1-inch wide (non-conductive) tape by using a passive reel with motorized pinch rollers to pay the tether out.

\section{Conclusion}

We have reviewed space applications of electrodynamic tethers, and basic issues and constraints on their operation. We reconsidered the bare-tether as an effective device solving the problem of electron collection from a rarefied magnetized plasma; thin-foil tethers that collect as much current than round-wire tethers prove to be much lighter. We have shown that for times at least moderately long, bare electrodynamic tethers are more mass-efficient than their direct competitor for deorbiting or reboost, the Ion Thruster. Also, in absence of solar power, a combined use of tether and chemical rocket is again more mass-efficient in power generation than direct use of fuel cells. We briefly recalled two other tether uses, Radation Bell Remediation and generation of electron beams for atmospheric research. Tether heating, arcing, and bowing or breaking, as well deployment strategies were discussed; very thin

a foils may present too much bowing unless they are made to spin, whereas they keep cold throughout. We note here that conditions used in the analysis correspond to Low Earth Orbit; conditions (much lower plasma density, much higher motional field) and conclusions for tethers in Jupiter can be quite different.

Acknowledgement

Work by J.R. Sanmartin was supported by the Spanish Ministerio de Ciencia y Tecnologia under Grant BFM013723.

\section{References}

${ }^{\mathrm{G}} \mathrm{Gross1,}$ M D, "Tether Hustory and Hustoriography", $2^{\text {nd }}$ International Conference on Tethers $m$ Space, Venice, Italy, 1987 , pp 3-8, Cosmo, M L and Lorenzin, E C , "Tethers in Space Handbook", Smithsonian Astrophysical Observatory, $3^{\text {td }}$ edition, Cambroge, MA, December 1997

${ }^{2}$ Johnson, L, Estes, RD, Lorenzim, E C , Martunez-Sanchez, M, and Sanmartın J R, "Propulsive Small Expendable Deployer System Experument" Journal of Spacecrafl and Rockets, Vol 37, No 2, 173-176, 2000

${ }^{3}$ Carrol, J A, "Tether Appl 1cations in Space Transportation", Acto Astronomica, Vo 13, 1986, pp 165-174

${ }^{4}$ Sanmartın, J R and Lorenzini, EC, "The role of superconductng shelds in electrodynamic propulsion", Joumal of Propulston and Power, July/August issue, 2008

Pelaez, J, Lorenzini, E C , Lopez-Rebolfal, $O$, and Runz, M , "A new kind of dynamic instability in electrodynamic tethers", Journal of Astronautcal Scjences, Vol 48, 2000, pp 449-476

"Wheelock, A, Cooke, D and Gess, M, "Initıal Plasma Tests of the IProSEC cathode device", 10" Spacecraft Charging Technology Conference, Blarritz, France, June 2007

${ }^{7}$ Sanmartın, J R, Martinez-Sanchez, M, and Ahedo, E, "Bare Wire Anodes for Electrodynamic Tethers," Journal of Propulsion and Power, Vol 9, No 3, 1993, pp 353-360

"Sanmartin, J R and Estes, R D, "The orbital-motion-limited regume of cylindncal Langtiuur probes", Physics of Flasmas, Vol 6, No 1, 1999, 395-405

"Estes, RD and Sanmartin, JR, "Cylundrical Langmuir probes beyond the orbatal-motion-limited regme", Physics of Plasmas, Vol 7, No 10, 2000, 4320-4325

${ }^{10}$ Chomère, $\mathrm{E}$ and Gilchrist, B "Self-Consistent 2-D Kunetıc Simulations of High-Voltage Plasma Sheaths Surrounding Ionattracting Conductive Cylinders in Flowng Plasmas", IEEE Transactions on Plasma Sctence, Vol 35, No 1, 2007, pp 7.22 
${ }^{14}$ Ferry, J B and Martinez-Sanchez, M, "Electron collection by a tether at high potential in a magnetızed plasma", Paper AIAA-3-4948, 39 $9^{\text {th }}$ Joint Proplusion Conference, Huntsville, AL, July 2003

${ }^{12}$ Sanmartin, J.R, Choinière, E, Gilchrist, B E , Ferry, J-B, and Martinez-Sánchez, M "Bare-Tether Sheath and Current Comparison of Asymptotıc Theory and Kinetic Simulations in a Stationary Plasma", IEEE Transactions on Plasma Sctence, (to appear)

${ }^{13}$ Laframboise, J.G and Parker, L W , "Probe design for orbt-limit current collection", The Physics of Fluds, Vol. 16, No. 5, 1973 , pp 629-636.

${ }^{14}$ Sanmartin, J.R, and Estes, RD, "Interference of parallel cylindrical Langmuur probes", Physscs of Plasmas, Vol. 8, No 9, 2001, pp. 4234-4239.

${ }^{13}$ Vannaron, $\mathrm{G}_{\text {, }}$ Dobrowolny, $\mathrm{M}$, and De Venuto, F, "Deorbitung of LEO Satellites with Electrodynamic Tethers", Paper AIAA 2000-0328, 38 th Aerospace Sciences Meetung and Exhbit, Reno, NV, Enero 2000

${ }^{16}$ Onishi, T, "Numerical Study of Current Collection by an Orbiting BareTether", PhD Thesis, MTT, August 2002; Deux, JM, Batischev, O V., and Martinez-Sanchez, M., "Advanced Kinetic Model for Electrodynamuc Space Tether", Proc. $4^{\text {th }}$ InternatınaI Space Propulsıon Conference, Caligari, Italy, June 2-4, 2004.

${ }^{17}$ Gilchrist, B.E, Bilen, S.G. and Chonère, E., "Analysis of Chamber Simulations of Long Collecting Probes in High Speed Dense Plasmas", IEEE Transactions on Plasma Scaence, Vol, 30, No. S, 2002, pp 2023-2034, Chounere, E., Blen, S G, Gilchrist, B E, Furhop, K.R, and Gallımore, A.D., "Experimental Investigation of Electron Collection to Solid and Slotted Tape Probes in a High-Speed Flowing Plasma", IEEE Transactons on Plasma Sctence, Vol, 33, No. 8, 20015, pp 1310-1323.

${ }^{18}$ Fujii, H.A., Takegahara, H, Dyama, K, Sasaki, S, Yamagiwa, Y, Krujff, M., Van der Helde, E J, Sanmartin, J.R, and Charro, M., "A proposed bare-tether experiment on board a sounding rocket", Paper ALA 2004-5718, 2 ad International Energy Conversion Engineermg Conference, 16-19 August, Providence, RI, 2004.

${ }^{19}$ Khazanov, G.V , Stone, N.H., Krivorutsky, EN, and Liemohn, M.W., "Current-produced magnetic field effects on current collection", Journal of Geophysscal Research, Vol 105, No A7, 2000, pp 15,835-15,842; Sanmartin, J R, and Estes, R.D., "Magnetic self-field effects on current collection by an ionospheric bare tether", Journal of Geophysical Research, Vol. 107, No. A11, 2002, SIA 2-1/2-7

${ }^{20} \mathrm{Ahedo}, \mathrm{E}$, and Sanmartm, J R, "Analysıs of Bare-Tether Systems for Deorbiting Low-Earth-Orbit ", Journal of Spacecraft and Rockets, Vol. 39, No 2, 2002, pp. 198-205

${ }^{2 !}$ Sanmartir, J.R., Estes, R D, Lorenzini, E.C , and Elaskar, S.A., "Efficiency of Electrodynamuc Tether Thrusters", Journal of Spacecraft and Rockets, Vol. 43, No. 3, 2006, pp. 659666

${ }^{22}$ Estes, R D., Lorenzini, E C, Sanmartm, J.R, Pelaez, J, Martinez-Sanchez, M, and Johnson L "Bare Tethers for Electrodynamic Spacecraft Propulsion", Journal of Spacecraft and Rockets, Vol. 37, No. 2, 2000, pp 205-211

${ }^{23}$ Martinez-Sanchez, M and Hastings, D, "A Systems Study of a $100 \mathrm{~kW}$ Electrodynamic Tether", The Journal of the Astronatical Sctences, Vol 35, No 1, 1987, pp 75-96.

${ }^{24}$ Sanmartin, J R, "Physıcs and applicatıons of electrodynamic space tethers", in Simplicity, Rigor and Relevance m Flutd Mechancs / A volume in honor of Amable Liñon, eds F.J. Higrera, J. Jmenez and J M Vega, CIMNE, Barcelona, Spain, 2004.

${ }^{25}$ Dantlov, V V., Elgin, B.A , Grafodatsky, OS and Mirnov, V V, "Hıgh-Voltage Satellite Tethers for Active Experiments m Space", $6^{\text {th }}$ Spacecraft Charging Technology Conference, AFRL-VS-TR-20001578, September 2000.

${ }^{26}$ "Zeineh, C, "Application of an Electrostatic High Voltage Tether to Radation Belt Remediation", M.S. Thesis, Mri, 2005

${ }^{27}$ Ashenberg, $\mathrm{J}$ and Lorenzini, E C., "Dynamics of a Dual-Probe Tethered System", Journal of Guadance, Control and Dynamics, Vol 20, No 6, 1997, pp 1265-1268

${ }^{28}$ Martinez-Sanchez, $M$ and Sanmartin, JR, "Artificial auroral effects form a bare conducting tether", Journal of Geophysical Research, Vol. 102, 1997, pp. 27,257-27,263, Sanmartın, J.R., Charro, M., Pelaez, J., Ttrao, I., Elaskar, S, Hilgers, A and Martinez-Sanchez, M., "Floating bare tether as upper atmosphere probe", Journal of Geophysical Research, Vol. 111, Al 1310,2006

${ }^{29}$ Vaughn, J.A, Curtss, L and Welzyn, K J "Plasme Interactions with a Negative Biased Electrodynamic Tether", $8^{\text {th }}$ Spacecraft Charging Technology Conference, Huntsville, AL, October 2003.

${ }^{30}$ Cosmo, ML, Lorenzin, E.C, and Bombardelli, C, "Space Tethers as Testbeds for Spacecraft Formation-Flying" Proceedings of the $14^{\text {th }}$ AAS/AIAA Space Flight Mechantcs Conference, Paper AAS 04-171, Maui, Hawall, February 2004

${ }^{31}$ Koss, S , "Tether deployment mechantsm for the advanced tether experment (Atex)." Procs. of the $7^{\text {th }}$ European Space Mechantsm and Tribology Sympostum, ESTEC Noordwijk, The Netherlands, ESA SP-410, October 1997 